\title{
BMJ Open Heart Watch Study: protocol for a pragmatic randomised controlled trial
}

\author{
Sanket S Dhruva (D) , , Nilay D Shah,, ${ }^{3,4}$ Sreekanth Vemulapalli, \\ Abhishek Deshmukh, ${ }^{7}$ Alexis L Beatty, ${ }^{2,8}$ Ginger M Gamble (i) ,9 \\ James V Freeman, ${ }^{9,10}$ James P Hummel, ${ }^{10}$ Jonathan P Piccini (i) , 5,6 \\ Joseph G Akar, ${ }^{10}$ Keondae Ervin, ${ }^{11}$ Kristine L Arges, ${ }^{5,6}$ Lindsay Emanuel, ${ }^{3,4}$ \\ Peter A Noseworthy, ${ }^{4,7}$ Tiffany Hu, ${ }^{9}$ Victoria Bartlett, ${ }^{12}$ Joseph S Ross ${ }^{9,13}$
}

To cite: Dhruva SS, Shah ND, Vemulapalli S, et al. Heart Watch Study: protocol for a pragmatic randomised controlled trial. BMJ Open 2021;11:e054550. doi:10.1136/ bmjopen-2021-054550

- Prepublication history and additional supplemental material for this paper are available online. To view these files, please visit the journal online (http://dx.doi.org/10.1136/ bmjopen-2021-054550).

Received 16 June 2021 Accepted 03 December 2021

Check for updates

(C) Author(s) (or their employer(s)) 2021. Re-use permitted under CC BY-NC. No commercial re-use. See rights and permissions. Published by BMJ.

For numbered affiliations see end of article.

Correspondence to Dr Sanket S Dhruva; sanket.dhruva@ucsf.edu

\section{ABSTRACT}

Introduction Personal digital devices that provide health information, such as the Apple Watch, have developed an increasing array of cardiopulmonary tracking features which have received regulatory clearance and are directly marketed to consumers. Despite their widespread and increasing use, data about the impact of personal digital device use on patient-reported outcomes and healthcare utilisation are sparse. Among a population of patients with atrial fibrillation and/or atrial flutter undergoing cardioversion, our primary aim is to determine the impact of the heart rate measurement, irregular rhythm notification, and ECG features of the Apple Watch on quality of life and healthcare utilisation.

Methods and analysis We are conducting a prospective, open-label multicentre pragmatic randomised clinical trial, leveraging a unique patient-centred health data sharing platform for enrolment and follow-up. A total of 150 patients undergoing cardioversion for atrial fibrillation or atrial flutter will be randomised 1:1 to receive the Apple Watch Series 6 or Withings Move at the time of cardioversion. The primary outcome is the difference in the Atrial Fibrillation Effect on QualiTy-of-life global score at 6 months postcardioversion. Secondary outcomes include inpatient and outpatient healthcare utilisation. Additional secondary outcomes include a comparison of the Apple Watch ECG and pulse oximeter features with gold-standard data obtained in routine clinical care settings.

Ethics and dissemination The Institutional Review Boards at Yale University, Mayo Clinic, and Duke University Health System have approved the trial protocol. This trial will provide important data to policymakers, clinicians and patients about the impact of the heart rate, irregular rhythm notification, and ECG features of widely used personal digital devices on patient quality of life and healthcare utilisation. Findings will be disseminated to study participants, at professional society meetings and in peer-reviewed journals.

Trial registration number NCT04468321

\section{INTRODUCTION}

The pace of innovation for digital health technologies is accelerating. Many individuals now have access to personal digital devices that track activity, sleep and weight. Some devices also monitor physiological
Strengths and limitations of this study

- This randomised clinical trial uses a patient-centred health data sharing platform for study enrolment and follow-up, leveraging patient ownership over health data and patient engagement with researchers.

- This is the first study to examine the impact of ECG and irregular rhythm notification features of personal digital technologies on patient-reported qualityof-life among patients with atrial fibrillation and/or atrial flutter.

- Due to costs of personal digital devices, our study is powered to identify a difference of 8.8 on the primary outcome of Atrial Fibrillation Effect on QualiTy-oflife global score, which is greater than the minimal clinically important difference of 5 .

- Our study focuses on a subset of patients with atrial fibrillation and/or atrial flutter, and the findings may not be generalisable to those who do not have these cardiac arrhythmias.

measures such as oxygenation, heart rate and rhythm, and blood pressure. ${ }^{1-3}$ In response, the US Food and Drug Administration (FDA) is working to establish a least burdensome regulatory framework for such technologies under its Digital Health Innovation Action Plan. ${ }^{4}$ For software-based medical devices, the agency has initiated a Software Precertification (Pre-Cert) Pilot; a key element is collecting and interpreting post-market, realworld data about software performance. ${ }^{6}$ In September 2018, FDA cleared two software features for the Apple Watch (Apple, Cupertino, California, USA) through the De Novo medical device regulatory pathway: (1) to detect irregular heart rhythms likely to be atrial fibrillation ${ }^{7}$ and (2) to generate a singlelead electrocardiogram (ECG) ${ }^{8}$ However, several risks were identified, including: misinterpretation and/or over-reliance on the device, false negative results and false positive results. Strategies to mitigate these risks were also identified. ${ }^{78}$ Additionally, multiple other 
digital devices have come to market that can perform ECGs and offer irregular rhythm notifications; devices with these features are among the most commonly used in digital health. Since that time, Apple has released an oxygen saturation monitor, which was not cleared by the FDA, as well as an updated feature for detection of irregular heart rhythms that received $510(\mathrm{k})$ regulatory clearance. ${ }^{9}$

Catalysed by the COVID-19 pandemic, these digital health monitoring technologies have been rapidly integrated into clinical practice to support patient monitoring in lieu of in-person visits, thereby reducing possible patient and clinician exposure. ${ }^{10}$ Insurers also sometimes provide coverage for these devices. ${ }^{11}$ These devices have inverted the traditional pyramid of scientific discovery. ${ }^{12}$ Medical products (ie, traditional new medical devices and drugs) are generally discovered and tested through scientific research that leads to regulatory approval and is disseminated to clinicians, who in turn inform patients of tests and treatments authorised for commercial use. While traditional medical devices and drugs can be marketed directly to patients, clinician prescriptions or clinical care are necessary for patient use.

In contrast, personal digital devices follow a different path. Many (or all) health and wellness-focused features are not reviewed or regulated by the FDA, while others are cleared if they are similar to previously cleared or approved medical devices. They are directly marketed to the public by manufacturers; patients consume marketing information and may purchase and use the devices without clinician oversight —or any direct interaction with the medical community. Patients may approach clinicians about using personal digital devices, but clinicians may not have scientific research available to guide them. ${ }^{12}$ This issue occurs with the Apple Watch, which is widely advertised and now exceeds 30 million units in annual sales. However, there are limited data on the impact of commonly used health features such as heart rate measurement, irregular rhythm notification and ECG features on patient quality of life ${ }^{13}$ and healthcare utilisation. Further, there have been limited evaluations of the accuracy of more novel health features such as the single lead ECG and pulse oximeter features.

Therefore, there is a need to assess the clinical impact of these devices through active surveillance to guide future labelling and risk mitigation strategies. While the Apple Heart Study reported positive predictive values for the irregular pulse notification ${ }^{2}$ and a subsequent study of cardiac surgery patients provided data on accuracy of atrial fibrillation detection, ${ }^{14}$ it is unknown how such notifications affect patients. There has been concern expressed about smartwatches leading to patient anxiety. ${ }^{15}$ In fact, anxiety disorders associated with these devices have been documented. ${ }^{16}$ Further, there are limited data about the impact on healthcare utilisation of Apple Watch heart rate, irregular rhythm notification, and ECG features. One single-health system study found higher overall healthcare utilisation among patients with atrial fibrillation using personal digital devices, including the Apple Watch, as documented in clinical notes compared with a propensity-matched sample of patients without documentation of these devices; the authors concluded by calling for prospective, longitudinal, randomised data on health outcomes and healthcare use. ${ }^{17}$ Some clinicians have expressed worry about cascades of testing to confirm or rule out disease. ${ }^{15}$ One single-centre study found that only $11.4 \%$ of patients had a clinically actionable diagnosis of interest after an irregular rhythm notification, although several tests (including Holter monitors, echocardiograms and even CT scans of the chest) were performed. ${ }^{18}$ Additionally, a small study has raised the possibility that Apple Watch heart rate measurements among patients with atrial fibrillation may sometimes be inaccurate $^{19}$; this is a known limitation of photoplethysmography-based heart rate monitors, given pulse deficit can occur in atrial fibrillation. ${ }^{1}$ Therefore, further validation is needed. ${ }^{19}$

Finally, there is a need to evaluate the accuracy of the newer ECG and pulse oximeter features. The ECG feature has been compared with 12-lead ECGs in two studies, although one focused only on the QT interval in sinus rhythm ${ }^{20}$ and another included only healthy volunteers. ${ }^{21}$ There are no published data about the accuracy of the oxygen saturation monitor.

Accordingly, we designed the Heart Watch Study, a pragmatic randomised controlled trial (RCT), to address these issues. We focus on atrial fibrillation, as it is the most common cardiac arrhythmia, affecting approximately 38 million people ${ }^{22}$ and may lead to adverse symptoms such as fatigue, palpitations and dyspnoea, ${ }^{23}$ and atrial flutter, which is a related and commonly co-occurring arrhythmia. This trial will provide an independent assessment of the impact of the Apple Watch heart rate, irregular rhythm notification and ECG features on (1) quality of life and (2) healthcare utilisation, as well as provide information about accuracy of measurements among patients undergoing cardioversion for atrial fibrillation or atrial flutter at three geographically distinct academic medical centres in the USA. By comparing the Apple Watch to a personal digital device without the ability to perform an ECG, offer irregular rhythm notifications, or measure pulse oximetry, we seek to better understand the impact of these health features of the Apple Watch. In addition to informing physicians, patients and policy-makers, these data can inform future labelling and risk mitigation strategies that could impact millions of individuals.

\section{OVERALL STUDY DESIGN}

Heart Watch is a prospective, open-label multicentre pragmatic RCT designed to evaluate the impact of patient use of the Apple Watch, focused on its accompanying health features of heart rate measurement, irregular rhythm notification and ECG features on patient-reported outcomes and healthcare utilisation at 6 months. Patients 


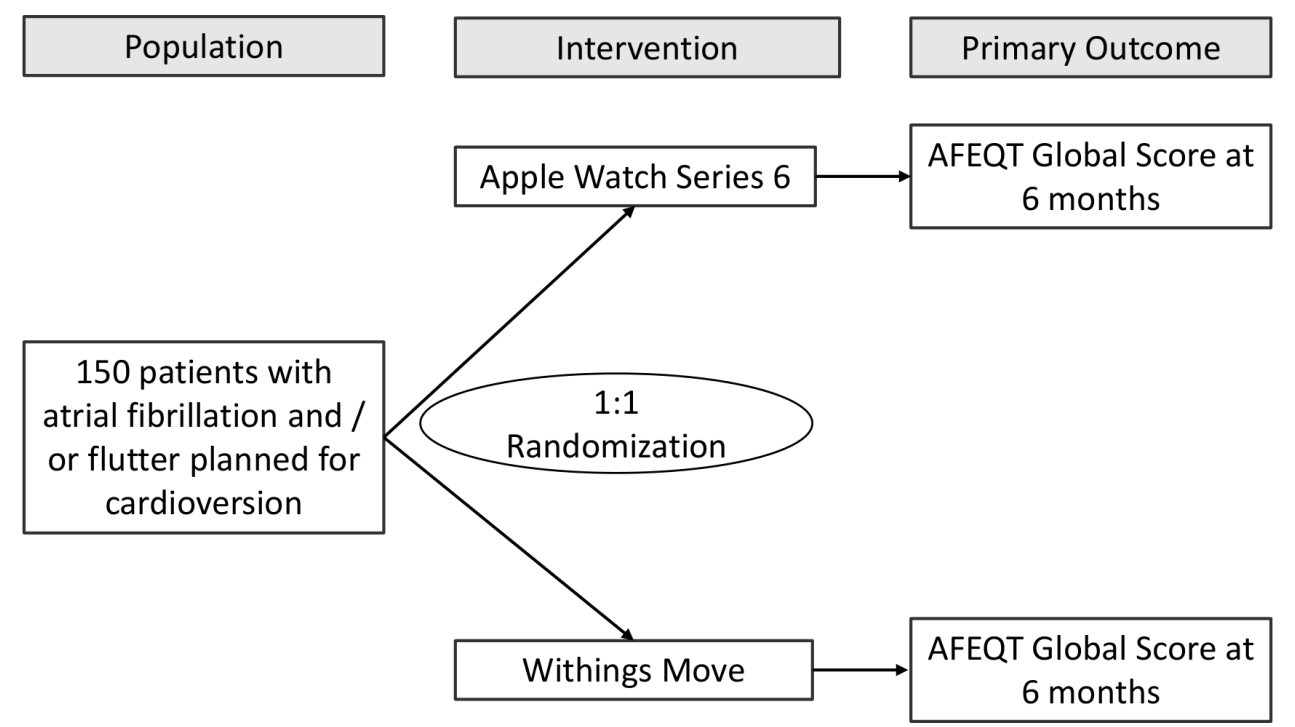

Figure 1 Study flow diagram. AFEQT, Atrial Fibrillation Effect on QualiTy-of-life.

with a history of atrial fibrillation and/or atrial flutter who are scheduled for cardioversion are randomised 1:1 to receive the Apple Watch Series 6 or the Withings Move (figure 1). The initial patient population of interest was patients with atrial fibrillation; however, in response to slower than anticipated enrolment, we revised our study protocol to include patients with atrial flutter. The study began with patient screening for enrolment in July 2021 with anticipated completion by 31 December 2022.

\section{DATA ACQUISITION}

Heart Watch leverages a unique patient-centred health data sharing platform, Hugo, which is intended to overcome many of the limitations of traditional clinical trials. ${ }^{24}$ Hugo aggregates electronic health data for each patient from multiple sources, including electronic health records from hospitals and physicians' offices, along with data from personal digital devices, by leveraging Blue
Button technology and Application Programming Interfaces (figure 2). Blue Button technology refers to functionality that enables individuals to download their health records. ${ }^{25}$ Hugo also supports direct patient surveys through text messaging or emails, enabling assessment of patient-reported outcomes and other information without requiring patients to meet directly with study coordinators after initial enrolment. All participants will be enrolled in this platform, through which they will receive near real-time access to their electronic health data from these multiple sources and then share these data with researchers. ${ }^{26}$ No data, therefore, are directly obtained from health systems.

\section{SAMPLE SELECTION}

A total of 150 patients will be enrolled at one of three academic health systems in equal numbers $(50$ at each health system): Yale New Haven Health, Mayo Clinic,

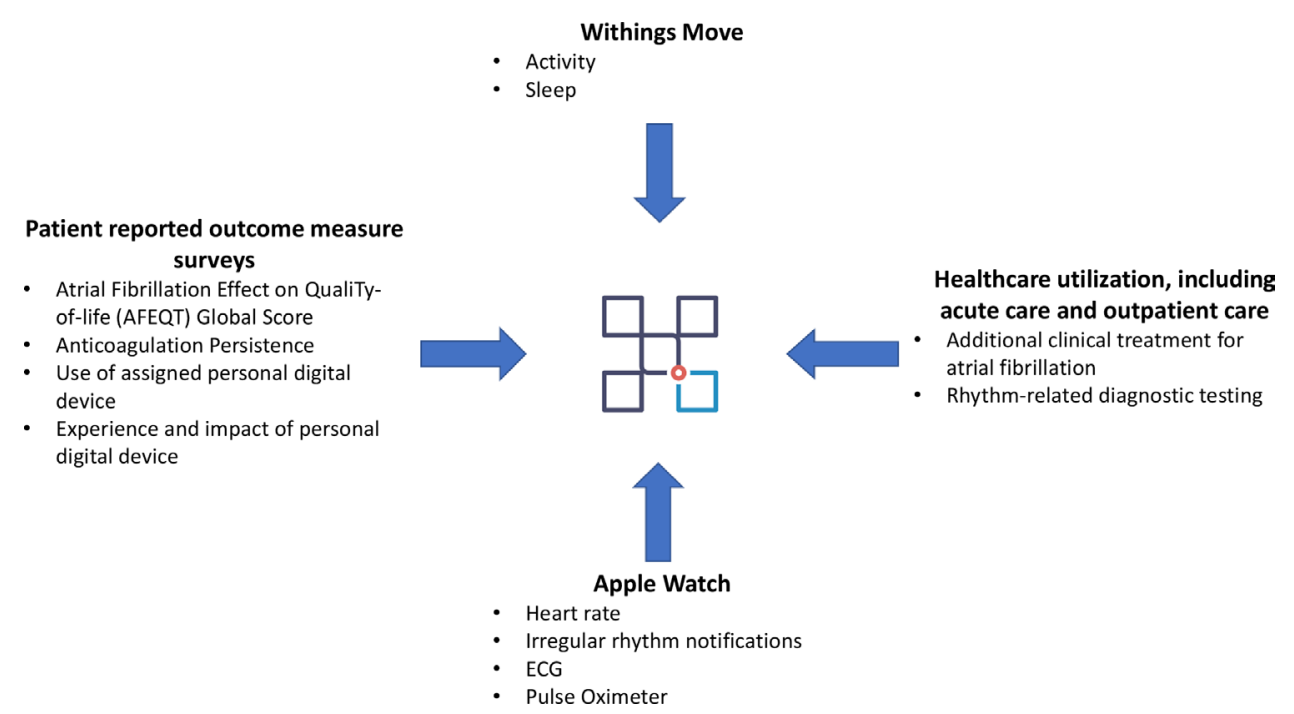

Figure 2 Data aggregated using the Hugo platform. 


\section{Box 1 Inclusion and exclusion criteria}

Inclusion criteria
Age $>22$ years at the time of enrolment
English-speaking
Planned for direct current cardioversion for atrial fibrillation or atrial
flutter
Lives independently and does not require continuous care
Has an email account (or is willing to create one)
Has a compatible smartphone (iPhone 6 s or later)
Willing to wear only the device to which they are randomised to receive
for the study period for as many hours during the day as able, except
for time spent charging the device or in environments that may be sub-
optimal for the device
Willing to use the Hugo Health platform
Receives cardiology care at one of the study centres
Receives primary care at a health system that can be linked to the Hugo
Health platform
Exclusion criteria
Not able to independently adhere to study protocol, including read and
sign consent
Enrolment in another study protocol

and Duke Health. Potential patients are identified by members of the clinical care team if they have been referred for cardioversion for atrial fibrillation or atrial flutter. If a patient in the outpatient setting expresses interest in the study and meets inclusion criteria of having an iPhone and receiving cardiology care at one of the academic health systems, then a research team member follows up with them to verify additional inclusion criteria and asks the patient to arrive early for enrolment prior to their cardioversion. We had initially planned to enrol only patients with both primary and cardiology care at one of the three health systems; however, in response to slower than anticipated enrolment, we revised our protocol to include patients with primary care outside of the academic health systems. These patients will still be required to have digital access to their primary care health data outside of the academic health systems. Patient identification varies slightly by site, but essentially hospitalised patients planned for cardioversion are identified prior to their procedure and, if they meet inclusion criteria, offered the opportunity to enrol.

\section{INCLUSION AND EXCLUSION CRITERIA}

Inclusion criteria are confirmed prior to the informed consent process and documented (box 1). Patients who do not meet all inclusion criteria or who meet any of the exclusion criteria do not proceed with consent and enrolment. Study coordinators obtain informed consent, and patients will sign a digital consent form (online supplemental material 1).

\section{INTERVENTIONS}

After signing informed consent documentation, patients are randomised 1:1 to the Apple Watch Series 6 (Global
Positioning System [GPS]) or Withings Move arm using a central computer-generated randomisation algorithm that is stratified by site to ensure balance. We pre-specified that each of the three clinical sites will enrol a total of approximately 25 patients in each arm.

The Apple Watch Series 6 is a smartwatch manufactured by Apple (Cupertino) that can obtain multiple physiologic measurements, including activity, sleep and several cardiopulmonary parameters. Activity features include steps taken, flights of stairs climbed, walking and running distance, energy expenditure and data about time spent standing. Cardiac features include heart rate, both at rest and with activity, and heart rate variability. Additionally, the Apple Watch offers optional notifications for low and high heart rates and irregular rhythms. The triggers for low and high heart rate notifications can be customised by the user. The device also allows users to perform a single-lead ECG and pulse oximetry measurement. The Withings Move is a smartwatch manufactured by Withings (France) that enables tracking of activity and sleep. Activity features include steps taken, distance travelled and energy expenditure. Sleep features include details about light and deep sleep phases, as well as sleep interruptions.

For this study, all data are confidentially aggregated using Hugo. Patients randomised to the Apple Watch arm link their Apple HealthKit account to the Hugo platform, which enables receipt of Apple Watch-related data. Patients randomised to the Withings Move share data using a Withings Health Mate account, which is also linked to the Hugo platform. Research coordinators at each clinical site assist patients with creating and linking these accounts. Patients who would like to do so can share their pharmacy data and Medicare claims data. Patientreported outcome measure $(\mathrm{PROM})$ questionnaires are texted or emailed to patients (depending on patient choice), enabling patients to answer queries using a secure link. Patients are reminded to wear their personal digital device and share data, as well as to complete PROM questionnaires, in order to improve adherence and response rates. Patients may discontinue study participation at any time during the study.

\section{COMPENSATION}

All patients will be able to keep the personal digital device to which they have been randomised: the Apple Watch Series 6 (GPS), with fair market value US\$399 in mid-2021 and the Withings Move with fair market value US $\$ 70$. Additionally, patients randomised to the Withings Move receive a stipend for time contributed to study participation and survey completion because of the lower cost of this device. The estimated hourly stipend is based on the average minimum wage across the recruitment sites (approximately US\$10), resulting in a total US\$80 stipend. Patients receive payments every 30 days on completing surveys; these payments are provided as a digital Visa card. 


\begin{tabular}{ll}
\hline $\begin{array}{l}\text { Table } 1 \\
\text { baseline from patient self-report }\end{array}$ & \multicolumn{1}{l}{ Demographic and clinical variables obtained at } \\
\hline Demographics & Clinical comorbidities \\
\hline Age & Hypertension, also called high blood pressure \\
\hline Sex & High cholesterol \\
Race & Coronary heart disease \\
Ethnicity & $\begin{array}{l}\text { A heart attack (also called myocardial } \\
\text { infarction) }\end{array}$ \\
& A stroke or transient ischaemic attack \\
& Emphysema \\
& Asthma \\
& Cancer or a malignancy of any kind \\
& Diabetes \\
& Weak or failing kidneys \\
& Any liver condition \\
& Post-traumatic stress disorder \\
& Depression \\
& General anxiety disorder \\
\end{tabular}

\section{PATIENT AND PUBLIC INVOLVEMENT}

The development of the research question and outcome measures were informed by the increasing consumer use of personal digital devices that provide health information, but with an absence of evidence about patient experience. Patients and the public were not involved in study design. Study results will be shared with study participants through an email summary after peer-reviewed publication and a patient facing summary will be prepared and disseminated via our funder and collaborative partner, the National Evaluation System for health Technology Coordinating Centre (NESTcc).

\section{DEMOGRAPHIC AND CLINICAL CHARACTERISTICS}

Demographic characteristics collected at enrolment include age, sex, race and ethnicity through patient self-report with discrete response categories (table 1). Patients also self-report the presence of multiple cardiovascular and non-cardiovascular comorbidities through a digital questionnaire at enrolment.

\section{OUTCOMES}

\section{Primary outcome}

Nearly all data elements for outcomes are aggregated through the Hugo platform. The primary outcome is the difference in the Atrial Fibrillation Effect on QualiTy-oflife (AFEQT) questionnaire ${ }^{27}$ global score at 6 months compared with baseline. The AFEQT questionnaire is a validated, 20-item questionnaire that has been shown to reliably track clinical changes in patients with atrial fibrillation. It contains two sections. The first section asks about the last episode of atrial fibrillation (one question). The second section contains 19 questions in four domains: symptoms (four items), daily activities (eight items), treatment concern (six items) and treatment satisfaction (one item). A composite overall score that ranges from 0 to 100 is calculated from the symptoms, daily activities, and treatment concern domains; higher values suggest better quality of life. Each domain also has a corresponding subscale score that also ranges from 0 to 100 . Published data indicate that a change of approximately five in the overall score indicates a clinically meaningful difference. ${ }^{28}$ We modified the AFEQT questionnaire into a digital format.

\section{Secondary outcomes}

The secondary outcomes can be divided into three distinct groups (table 2). The first group of secondary outcomes includes five patient-reported outcomes. First, we will examine the change in AFEQT global score at 1 , 2, 3, 4, 5 and 12 months (the primary outcome examines this score at 6 months); this will provide additional insight into changes in the primary outcome. Second, at all time points we will examine the change within individual domains of the AFEQT questionnaire (symptoms, daily activities, treatment concern and treatment satisfaction); this will allow us to determine if the personal digital devices have different effects on specific patientreported outcomes. Third, we ask about anticoagulation persistence at 1, 2, 3, 4, 5 and 6 months and again at 12 months, since clinical practice guidelines recommend that patients receiving cardioversion should be on therapeutic anticoagulation for at least 4 weeks postcardioversion, ${ }^{23}$ and often patients meet criteria for longer or indefinite anticoagulation; we determine this through adopting the Brief Medication Questionnaire and asking patients if they have taken their anticoagulant, and for how many days in the past 1 week. Fourth, we ask patients at 1, 2, 3, 4, 5 and 6 months and again at 12 months if they have stopped using the personal digital device to which they were assigned (and, if so, the reason) as well as if they have used a different personal digital device (and which device). Fifth, we ask patients at 1, 2, 3, 4, 5 and 6 months and again at 12 months if they received any abnormal or worrisome notifications from their wearable device and, if so, if they contacted their physician and the result of the contact. Among patients randomised to the Apple Watch, we also ask if they have used the device to examine heart rhythm for symptoms. Sixth, we ask at 6 and 12 months about patient experience with technology; reassurance or worry about overall health; reassurance or worry about atrial fibrillation; and confidence about atrial fibrillation, physical activity and usual activities. Patients randomised to the Apple Watch arm are also asked about the amount of notifications.

The second group of secondary outcomes are healthcare utilisation outcomes. There are four healthcare utilisation outcomes, all of which seek to determine if patients randomised to the Apple Watch (with cardiopulmonary features) have different rates of obtaining care, including diagnostic testing and clinical treatment for atrial tachyarrhythmias, compared with patients receiving 
Table 2 Primary and secondary outcomes

\begin{tabular}{|c|c|c|c|c|c|c|c|c|}
\hline & & & & & & & & \\
\hline & Enrolment & 1 & 2 & 3 & 4 & 5 & 6 & 12 \\
\hline \multicolumn{9}{|l|}{ Primary outcome } \\
\hline \multicolumn{9}{|l|}{ Secondary outcomes } \\
\hline \multicolumn{9}{|c|}{ Patient-Reported Outcomes } \\
\hline $\begin{array}{l}\text { PRO about notifications } \\
\text { from device }\end{array}$ & & $\mathrm{x}$ & $\mathrm{x}$ & $\mathrm{x}$ & $x$ & $\mathrm{x}$ & $x$ & $x$ \\
\hline $\begin{array}{l}\text { PRO about use of assigned } \\
\text { personal digital device }\end{array}$ & & $\mathrm{x}$ & $\mathrm{x}$ & $\mathrm{x}$ & $x$ & $x$ & $\mathrm{x}$ & $x$ \\
\hline $\begin{array}{l}\text { PRO about experience and } \\
\text { impact of personal digital } \\
\text { device }\end{array}$ & & & & & & & $\mathrm{x}$ & $x$ \\
\hline $\begin{array}{l}\text { Additional clinical treatment } \\
\text { for atrial fibrillation or } \\
\text { flutter, such as repeat } \\
\text { cardioversion or catheter } \\
\text { ablation }\end{array}$ & & & & & & & $\mathrm{x}$ & $x$ \\
\hline Acute care use & & & & & & & $x$ & $\mathrm{x}$ \\
\hline Outpatient care use & & & & & & & $x$ & $\mathrm{x}$ \\
\hline $\begin{array}{l}\text { Rhythm-related diagnostic } \\
\text { testing }\end{array}$ & & & & & & & $x$ & $\mathrm{x}$ \\
\hline \multicolumn{9}{|c|}{ Apple watch cardiopulmonary feature comparisons } \\
\hline $\begin{array}{l}\text { Total no of ECGs and } \\
\text { notifications }\end{array}$ & & & & & & & $x$ & $x$ \\
\hline
\end{tabular}

AFEQT, Atrial Fibrillation Effect on QualiTy-of-life; PRO, patient-reported outcome.

the Withings Move. First, we will determine if patients receive additional clinical treatment for atrial fibrillation or flutter at 6 and 12 months by examining a composite of rhythm control intervention, which is defined as at least one additional cardioversion, initiation of antiarrhythmic medical therapy, dose escalation of antiarrhythmic medication, change to another antiarrhythmic medication, or ablation for atrial fibrillation or atrial flutter. The medication data will be obtained primarily from electronic health record data. Second, we will compare acute care use, a composite of emergency department visits, observation stays and all hospitalisations. Third, we will compare outpatient care use, a composite of outpatient primary care visits, outpatient cardiology or cardiac electrophysiology visits, and scheduled telephone encounters. Fourth, we will compare rhythm-related diagnostic testing at 6 and 12 months, a composite of total ECGs and total outpatient heart rhythm monitors.

The third group of secondary outcomes are only determined among patients randomised to the Apple Watch. We will determine the total number of notifications for irregular rhythm, high and low heart rates. To address the knowledge gap about the accuracy of the Apple Watch ECG, we perform a comparison between each Apple Watch ECG (using 10s interval with highest resolution and free from artefact) vs 12-lead ECG obtained in routine clinical practice as close to simultaneous as possible, ideally within a maximum window of $30 \mathrm{~min}$ to the Apple Watch ECG. The parameters that will be compared include heart rhythm (identical to 12-lead ECG or different), rate (difference in beats per minute), and intervals: PR, QRS and QT (difference in milliseconds). These metrics include both interobserver and intraobserver reliability. The Apple Watch ECG is obtained by asking each patient to email their Apple Watch ECG(s) to a secure study team email address at each health system, while information on the number of ECGs will be aggregated through Hugo. Finally, we compare pulse oximeter readings from the Apple Watch Blood Oxygen measurement to a standard, medical grade pulse oximeter in clinical practice. The pulse oximeter data are obtained when a research coordinator is at the patient's bedside during or after enrolment and attaches a medical grade pulse oximeter; the values are entered by a patient into a survey when the research coordinator is present. 


\section{SAMPLE SIZE CALCULATION}

Our sample size was determined assuming $80 \%$ power to detect a differential change of 8.8 on the AFEQT questionnaire global score at 6 months, the primary outcome, with alpha 0.05. This effect size is greater than the expected minimal clinically important difference of $5 .^{28}$ This estimate accounts for an estimated drop-out rate of $8 \%$ with respect to non-completion of the AFEQT questionnaire at 6 months.

For the secondary endpoints focused on healthcare utilisation, we expect drop-out to be close to zero because patients are followed passively with minimal burden and receive cardiology care at the enrolling health system. Additionally, the Hugo platform enables patients to share data from other health systems. Therefore, even if a patient switches care to outside one of the health systems of interest, if he/she connects their new health system (as portal availability is legally required), comprehensive outcome ascertainment will remain possible.

Validation of Apple Watch ECG readings with a simultaneous 12-lead ECG in clinical practice will be performed for as many ECGs as qualify. Similarly, pulse oximeter readings are performed and will be validated against those measured using a clinical pulse oximeter whenever possible.

\section{DATA ANALYSIS PLAN}

Data quality and integrity checks will be performed. Baseline descriptive statistics will be reported for the overall sample, and for both the Withings and Apple Watch arms. These will include patient age, sex, race, ethnicity and the comorbidities collected. Baseline data will be compared by $\chi^{2}$ (or Fischer's exact) test for dichotomous/categorical variables and t-tests for continuous variables and the median test for non-parametric variables. Data on missing covariates will be missing and not imputed.

For the primary outcome, we will calculate the difference in the AFEQT global questionnaire score (which ranges from 0 to 100) at baseline and at 6 months, and compare between patients randomised to the two study arms using a t-test.

The secondary outcomes will use a t-test for comparison of the AFEQT global questionnaire score between baseline and at 12 months and a t-test also for the individual domains at 1, 2, 3, 4, 5, 6 and 12 months. The secondary healthcare utilisation outcomes are composites and will use a t-test or the Wilcoxon rank-sum test for comparison between the two study arms. For the ECG comparison, inter-observer and intra-observer agreement will be assessed using intraclass correlation coefficients. In an exploratory analysis, the readings on the clinical pulse oximeter and Apple Watch pulse oximetry feature will be compared.

If patient drop-out or loss to follow-up occurs, then we will carry forward the last PROM response. If an electronic health record data connection is lost for a study participant, we will only include the follow-up duration when data were available in the utilisation endpoint analyses. All $p$ values will be significant at $<0.05$ with two-sided inferential tests. All analyses will be conducted at Mayo Clinic and data analysts will be blinded to treatment assignment.

\section{ETHICS AND DISSEMINATION \\ Ethics approval}

The Heart Watch Study is sponsored by NESTcc. Ethics approval was obtained independently at each of the three health systems, including at Yale University on 2 August 2020; at Mayo Clinic on 3 March 2021; and at Duke University Health System on 4 June 2021. Any amendments to the protocol are first reviewed by each of the three local institutional review boards prior to implementation.

Serious adverse events are not expected in this study of consumer personal digital devices. However, if there are device-related adverse events, they will be reported to the FDA's Manufacturer and User Facility Device Experience database. A data safety monitoring board was not convened because the intervention is low risk.

The investigator team makes clear that any sync-able data, including PROMs, will not be reviewed in real-time by researchers and will not be provided to the clinical care team and, therefore, any adverse or severe symptoms should be reported directly by patients to their physician(s) or emergency room physicians as they would have in the normal course of care.

\section{Dissemination}

RCT results will be presented at both scientific meetings and submitted for publication to peer-reviewed journals.

\section{DISCUSSION}

The Heart Watch Study pragmatic RCT addresses important knowledge gaps about the impact of novel, widely used personal digital devices on quality of life and healthcare utilisation. Despite the widespread and increasing use of these devices for both consumer and clinical applications, few studies have assessed their impact on these important outcomes. Some of the features are also marketed as health and wellness, without a requirement for FDA clearance. To our knowledge, no prospective studies of these technologies focused on these endpoints have been pursued independent of the device manufacturers; additionally, manufacturers have not conducted many studies in these areas and may lack incentives to do so.

In addition to the data generated, there are several strengths to this study. First, the use of a controlled design helps to isolate the impact of the cardiopulmonary features (heart rate, ECG, irregular rhythm notification and pulse oximeter) from activity tracking. Second, this study employs a novel pragmatic digital paradigm of research in which patients partner with researchers to share their data; data do not come directly from traditional 
sources, such as health systems. This research paradigm is supported by the principle of individual agency over data. ${ }^{24}$ Third, recent policy changes by Medicare provide reimbursement for remote patient monitoring using personal digital devices and this study will provide information about the implications of these changes. ${ }^{29} 30$

This RCT should also be considered in the context of multiple possible limitations. First, the study is powered to identify a difference of 8.8 in the AFEQT global score, which is greater than the minimal clinically important difference of $5,{ }^{28}$ due to the high costs of the devices provided to patients and the budgetary constraints of the trial. Second, the PROMs have been digitally adapted. The AFEQT questionnaire has been validated in paper format. ${ }^{27}$ However, the vast majority of PROMs that are adapted to a digital format result in synchronous responses ${ }^{31}$ and this approach is more accurate than clinician documentation. ${ }^{32}$ Third, there is the possibility of incomplete ascertainment of events if patients use a different health system for care and do not connect these with Hugo. However, the Hugo platform allows patients to add data from multiple health systems (and other sources) at any time throughout the study, thereby facilitating complete clinical event ascertainment. Fourth, the irregular rhythm notification is not intended for use in individuals previously diagnosed with atrial fibrillation. However, the ECG feature is not recommended for users with arrhythmias except for atrial fibrillation and sinus rhythm (since the software can only distinguish these two heart rhythms). ${ }^{8}$ People with atrial fibrillation and/or atrial flutter may be more likely to purchase this device than others to monitor their rhythm and correlate with symptoms. Indeed, in the Apple Heart Study, nearly 20\% of first study visit participants reported a diagnosis of atrial fibrillation/flutter before enrolment, even though a history of atrial fibrillation was an exclusion criterion. ${ }^{2}$ Few digital health studies have enrolled patients with clinical morbidities to examine outcomes; most studies have instead focused on healthy volunteers. ${ }^{33}$ Our study will address this limitation. Additionally, a study examining the impact of the Apple Watch on patient-reported outcomes and healthcare utilisation requires an enriched population; otherwise, study duration would be many years with a population size multiple times larger than our trial, resulting in significant cost and delays in generating much-needed evidence.

\footnotetext{
Author affiliations

${ }^{1}$ Section of Cardiology, San Francisco Veterans Affairs Medical Center, San

Francisco, California, USA

${ }^{2}$ Department of Medicine, University of California, San Francisco School of Medicine, San Francisco, California, USA

${ }^{3}$ Department of Health Sciences Research, Mayo Clinic, Rochester, Minnesota, USA ${ }^{4}$ Robert D. and Patricia E. Kern Center for the Science of Health Care Delivery, Mayo Clinic, Rochester, Minnesota, USA

${ }^{5}$ Duke Clinical Research Institute, Durham, North Carolina, USA

${ }^{6}$ Division of Cardiology, Duke University Medical Center, Durham, North Carolina, USA

${ }^{7}$ Department of Cardiovascular Medicine, Mayo Clinic, Rochester, New York, USA
}

${ }^{8}$ Department of Epidemiology and Biostatistics, University of California San Francisco School of Medicine, San Francisco, California, USA

${ }^{9}$ Center for Outcomes Research and Evaluation, Yale New Haven Hospital, New Haven, Connecticut, USA

${ }^{10}$ Section of Cardiovascular Medicine, Department of Internal Medicine, Yale School of Medicine, New Haven, Connecticut, USA

${ }^{11}$ National Evaluation System for health Technology Coordinating Center (NESTcC), Medical Device Innovation Consortium, Arlington, Virginia, USA

${ }^{12}$ Yale School of Medicine, New Haven, Connecticut, USA

${ }^{13}$ Department of Internal Medicine, Yale School of Medicine, New Haven, Connecticut, USA

Acknowledgements We would like to thank Harlan M. Krumholz, MD, SM, for his guidance on the conception and design of this trial and Jessica Ritchie and Laura Ciaccio for their assistance in the design and implementation of this trial.

Contributors SSD, NDS, SV, AD, ALB, GMG, JVF, JPH, JPP, JGA, PAN and JSR designed the study. SSD and JSR wrote the first draft of the manuscript. GMG is the overall study coordinator and TH, KLA and LE are individual site study coordinators. All authors revised the manuscript and approved the final version of the submitted manuscript. The corresponding author attests that all listed authors meet authorship criteria and that no others meeting the criteria have been omitted. SSD acts as the guarantor.

Funding This project was supported by a research grant from the Medical Device Innovation Consortium (MDIC) as part of the National Evaluation System for health Technology (NEST), an initiative funded by the US Food and Drug Administration (FDA). While MDIC provided feedback on project conception and design, the organisation played no role in collection, management, analysis and interpretation of the data, nor preparation, review and approval of the manuscript. The research team, not the funder, made the decision to submit the manuscript for publication. Funding for this publication was made possible, in part, by the US Food and Drug Administration through grant 1U01FD006292-01.

Disclaimer Its contents are solely the responsibility of the authors and do not necessarily represent the official views nor the endorsements of the Department of Health and Human Services or the FDA. Views expressed in written materials or publications do not necessarily reflect the official policies of the Department of Health and Human Services; nor does any mention of trade names, commercial practices or organisation imply endorsement by the United States Government

Competing interests SSD reports research funding from the National Heart, Lung, and Blood Institute (NHLBI, K12HL138046) of the National Institutes of Health (NIH), from the Medical Device Innovation Consortium (MDIC) as part of the National Evaluation System for health Technology Coordinating Center (NESTcC), Food and Drug Administration (FDA), Greenwall Foundation, and Arnold Ventures. NDS has received research support through Mayo Clinic from the FDA to establish the YaleMayo Clinic Center for Excellence in Regulatory Science and Innovation program (CERSI, U01FD005938), from the Centers of Medicare and Medicaid Innovation under the Transforming Clinical Practice Initiative, from the Agency for Healthcare Research and Quality (AHRQ, U19HS024075, R01HS025164, R01HS025402, R03HS025517), from the NHLBI of the NIH (R56HL130496, R01HL131535), from the National Science Foundation, and from the Patient Centered Outcomes Research Institute to develop a Clinical Data Research Network. SV reports receiving funding support from the American College of Cardiology, Society of Thoracic Surgeons, and the NIH (R01 and Small Business Innovation Research BIR), FDA (through NESTcc), Abbott and Boston Scientific. He reports serving as a consultant or on the Advisory Board of Janssen, HeartFlow, the American College of Physicians, and Boston Scientific. ALB reported former employment at Apple and holds stock in Apple Inc. JVF reported salary support from the American College of Cardiology National Cardiovascular Data Registry and the NHLBI and consulting/advisory board fees from Boston Scientific, Medtronic, and Biosense Webster. JPP reports receiving grants for clinical research from Abbott, American Heart Association, Association for the Advancement of Medical Instrumentation, Bayer, Boston Scientific, and Philips and serves as a consultant to Abbott, Abbvie, Ablacon, Altathera, ARCA Biopharma, Biotronik, Boston Scientific, Bristol Myers Squibb, LivaNova, Medtronic, Milestone, ElectroPhysiology Frontiers, Itamar, Pfizer, Sanofi, Philips, ResMed, and Up-to-Date. PAN receives research funding from NIH, including the NHLBI and the National Institute on Aging (NIA), AHRQ, FDA and the American Heart Association (AHA). He is a study investigator in an ablation trial sponsored by Medtronic. PAN and Mayo Clinic are involved in potential equity/royalty relationship with AliveCor. JSR received research support through Yale University from Johnson and Johnson to develop methods of clinical trial data sharing, from the FDA to establish YaleMayo Clinic CERSI program (U01FD005938), MDIC as part of the NESTcc, AHRQ 
(R01HS022882), NHLBI of the NIH (R01HS025164, R01HL144644), and from the Laura and John Arnold Foundation to establish the Good Pharma Scorecard at Bioethics International and to establish the Collaboration for Research Integrity and Transparency (CRIT) at Yale; in addition, JSR is an expert witness at the request of Relator's attorneys, the Greene Law Firm, in a qui tam suit alleging violations of the False Claims Act and Anti-Kickback Statute against Biogen Inc.

Patient consent for publication Not applicable.

Provenance and peer review Not commissioned; externally peer reviewed.

Supplemental material This content has been supplied by the author(s). It has not been vetted by BMJ Publishing Group Limited (BMJ) and may not have been peer-reviewed. Any opinions or recommendations discussed are solely those of the author(s) and are not endorsed by BMJ. BMJ disclaims all liability and responsibility arising from any reliance placed on the content. Where the content includes any translated material, BMJ does not warrant the accuracy and reliability of the translations (including but not limited to local regulations, clinical guidelines, terminology, drug names and drug dosages), and is not responsible for any error and/or omissions arising from translation and adaptation or otherwise.

Open access This is an open access article distributed in accordance with the Creative Commons Attribution Non Commercial (CC BY-NC 4.0) license, which permits others to distribute, remix, adapt, build upon this work non-commercially, and license their derivative works on different terms, provided the original work is properly cited, appropriate credit is given, any changes made indicated, and the use is non-commercial. See: http://creativecommons.org/licenses/by-nc/4.0/.

\section{ORCID iDs}

Sanket S Dhruva http://orcid.org/0000-0003-0674-2032

Ginger M Gamble http://orcid.org/0000-0002-8912-7440

Jonathan P Piccini http://orcid.org/0000-0003-0772-2404

\section{REFERENCES}

1 Bruining N, Caiani E, Chronaki C, et al. Acquisition and analysis of cardiovascular signals on smartphones: potential, pitfalls and perspectives: by the task force of the e-Cardiology Working Group of European Society of Cardiology. Eur J Prev Cardiol 2014;21:4-13.

2 Perez MV, Mahaffey KW, Hedlin H, et al. Large-scale assessment of a smartwatch to identify atrial fibrillation. $N$ Engl J Med 2019;381:1909-17.

3 Moon JH, Kang M-K, Choi C-E, et al. Validation of a wearable cuffless wristwatch-type blood pressure monitoring device. Sci Rep 2020;10:19015.

4 Food \& Drug Administration. Digital Health Innovation Action Plan, 2021.

5 Shuren J, Patel B, Gottlieb S. FDA regulation of mobile medical apps. JAMA 2018;320:337-8.

6 Food \& Drug Administration. Digital Health Software Precertification (Pre-Cert) program. Available: https://www.fda.gov/MedicalDevices/ DigitalHealth/DigitalHealthPreCertProgram/default.htm [Accessed June 4, 2021].

7 Food \& Drug Administration. CDRH clearance letter to Apple for irregular rhythm notification feature, 2018.

8 Food \& Drug Administration. CDRH clearance letter to Apple for ECG App, 2018.

9 Food and Drug Administration. Summary: Irregular Ryhthm Notification Feature (IRNF) 2.0 App. Available: https://www. accessdata.fda.gov/cdrh_docs/pdf21/K212516.pdf [Accessed November 22, 2021].

10 Varma N, Marrouche NF, Aguinaga L, et al. HRS/EHRA/APHRS/ LAHRS/ACC/AHA worldwide practice update for telehealth and arrhythmia monitoring during and after a pandemic. J Am Coll Cardiol 2020;76:1363-74.

11 Farr C. Private Medicare plan Devoted Health says it is the first to cover Apple Watch as a benefit, 2019. https://www.cnbc.com/2019/ 10/07/devoted-medicare-advantage-plan-covering-apple-watch-asa-benefit.html
12 Marcus GM. The Apple Watch can detect atrial fibrillation: so what now? Nat Rev Cardiol 2020;17:135-6.

13 Epstein $\mathrm{RH}$. Can a smartwatch save your life? The New York Times2021.

14 Seshadri DR, Bittel B, Browsky D, et al. Accuracy of Apple Watch for detection of atrial fibrillation. Circulation 2020;141:702-3.

15 Varshney AS, Madias C, Kakkar R, et al. Watching for disease: the changing paradigm of disease screening in the age of consumer health devices. J Gen Intern Med 2020;35:2173-5.

16 Rosman L, Gehi A, Lampert R. When smartwatches contribute to health anxiety in patients with atrial fibrillation. Cardiovasc Digit Health J 2020;1:9-10.

17 Wang L, Nielsen K, Goldberg J, et al. Association of wearable device use with pulse rate and health care use in adults with atrial fibrillation JAMA Netw Open 2021;4:e215821.

18 Wyatt KD, Poole LR, Mullan AF, et al. Clinical evaluation and diagnostic yield following evaluation of abnormal pulse detected using Apple Watch. J Am Med Inform Assoc 2020;27:1359-63.

19 Seshadri DR, Bittel B, Browsky D, et al. Accuracy of the Apple Watch 4 to measure heart rate in patients with atrial fibrillation. IEEE J Trans/ Eng Health Med 2020;8:1-4.

20 Strik M, Caillol T, Ramirez FD, et al. Validating QT-interval measurement using the Apple Watch ECG to enable remote monitoring during the COVID-19 pandemic. Circulation 2020;142:416-8.

21 Saghir N, Aggarwal A, Soneji N, et al. A comparison of manual electrocardiographic interval and waveform analysis in lead 1 of 12lead ECG and Apple Watch ECG: a validation study. Cardiovasc Digit Health J 2020;1:30-6.

22 Wang L, Ze F, Li J, et al. Trends of global burden of atrial fibrillation/flutter from Global Burden of Disease Study 2017. Heart 2021;107:881-7.

23 January CT, Wann LS, Alpert JS, et al. 2014 AHAVACC/HRS guideline for the management of patients with atrial fibrillation: a report of the American College of Cardiology/American Heart Association Task Force on Practice Guidelines and the Heart Rhythm Society. Circulation 2014;130:e199-267.

24 Dhruva S, Ross JS, Shah N. New federal rules pave the way for patient-driven health information exchange and real-world evidence on COVID-19 surveillance and treatment, 2020. Available: https://www.healthaffairs.org/do/10.1377/hblog20200506.368396/ full/

25 Mohsen MO, Aziz HA. The Blue Button project: engaging patients in healthcare by a click of a button. Perspect Health Inf Manag 2015;12:1d

26 Dhruva SS, Ross JS, Akar JG, et al. Aggregating multiple real-world data sources using a patient-centered health-data-sharing platform. NPJ Digit Med 2020;3:60.

27 Spertus J, Dorian P, Bubien R, et al. Development and validation of the Atrial Fibrillation Effect on Quality-of-Life (AFEQT) questionnaire in patients with atrial fibrillation. Circ Arrhythm Electrophysiol 2011;4:15-25.

28 Holmes DN, Piccini JP, Allen LA, et al. Defining clinically important difference in the atrial fibrillation effect on quality-of-life score. Circ Cardiovasc Qual Outcomes 2019;12:e005358.

29 Dey $\mathrm{P}$, Jarrin $\mathrm{R}$, Mori $\mathrm{M}$, et al. Leveraging remote physiologic monitoring in the COVID-19 pandemic to improve care after cardiovascular hospitalizations. Circ Cardiovasc Qual Outcomes 2021;14:e007618.

30 Mecklai K, Smith N, Stern AD, et al. Remote Patient Monitoring Overdue or Overused? N Engl J Med 2021;384:1384-6.

31 Muehlhausen W, Doll H, Quadri N, et al. Equivalence of electronic and paper administration of patient-reported outcome measures: a systematic review and meta-analysis of studies conducted between 2007 and 2013. Health Qual Life Outcomes 2015;13:167.

32 Pakhomov SV, Jacobsen SJ, Chute CG. Agreement between patientreported symptoms and their documentation in the medical record. Am J Manag Care 2008;14:530-9.

33 Safavi K, Mathews SC, Bates DW, et al. Top-funded digital health companies and their impact on high-burden, high-cost conditions. Health Aff 2019;38:115-23. 